\title{
Relaxation behaviour of gasketed joints during assembly using finite element analysis
}

\author{
MUHAMMAD ABID* and SAAD HUSSAIN \\ Faculty of Mechanical Engineering, Ghulam Ishaq Khan (GIK) Institute of \\ Engineering Sciences and Technology, Topi-NWFP 23460, Pakistan \\ e-mail: abid@giki.edu.pk
}

MS received 27 February 2008; revised 25 June 2009; accepted 30 November 2009

\begin{abstract}
Gasketed bolted flange pipe joints are always prone to leakage during operating conditions. Therefore, performance of a gasketed flange joint is very much dependent on the proper joint assembly with proper gasket, proper gasket seating stress and proper pre-loading in the bolts of a joint. For a gasketed flange joint, the two main concerns are the joint strength and the sealing capability. To investigate these, a detailed three-dimensional nonlinear finite element analysis of a gasketed joint is carried out using gasket as a solid plate. Bolt scatter, bolt bending and bolt relaxation are concluded as the main factors affecting the joint's performance. In addition, the importance of proper bolt tightening sequence, number of passes influence of elastic and elasto-plastic material modelling on joint performance are also presented. A dynamic mode in a gasketed joint is concluded, which is the main reason for its failure.
\end{abstract}

Keywords. Bolt; relaxataion; gasketed; joint; dynamic; tightening; sequence; solid; gasket.

\section{Introduction}

Gasketed pipe flange joints are widely used in industry to connect pipe to pipe or pipe to equipment. These are used in a wide variety of different applications from water supply to high pressure and high temperature applications. In a gasketed pipe flange joint, problem of bolt scatter, bolt bending and joint relaxation is observed; resulting in the dynamic mode-of-load (Abid \& Nash 2006; Abid 2000). Dynamic mode here represents a situation where the flange faces move and rotate relative to one another resulting in a change in the bolt load during operation. Such situation occurs when a gasket element is present. Due to this gasketed joints are prone to leakage, even after careful pre-loading and even for poor joint strength. A static mode is defined as no significant movement of the flange faces with a change in bolt load occurs. Some experimental and numerical investigations are performed to estimate bolt pre-load scatter due to the elastic interaction in the process of successive

*For correspondence 
bolt tightening (Fukuoka \& Takaki 2001, 2003). These investigations are limited to the linear elastic material modelling. In addition, these do not consider bolt bending behaviour, flange rotation and flange stress variation. Detailed experimental studies are performed to highlight bolt-bending behaviour, flange stress variation and flange rotation with special emphasis on the joint strength and sealing capabilities (Abid \& Nash 2006; Abid 2000). In the present study, a detailed three-dimensional nonlinear finite element analysis of a gasketed joint is carried out using gasket configurations as a solid plate. This is considered as gasket sealing portion and is completely compressed at the seating stress load applied during joint tightening. Bolt scatter, bolt bending, joint relaxation and gasket stress variation are concluded the main factors affecting the joint's performance. In addition, the importance of proper bolt tightening sequence, number of passes, influence of elastic and elasto-plastic material modelling on the joint performance are also presented. A flange joint of four-inch $900^{\#}$ class is used in the present study.

\section{Finite element analysis (FEA)}

\subsection{Modelling}

Abid \& Nash (2006) and Abid \& Baseer (2007) investigated joint strength and sealing capability under combined loading for an axi-symmetric 3-D model where the pre-load of each bolt was the same using a solid plate gasket. In this study, a complete 360 degree FE model was developed to study the effect of each bolt tightening on others. An angular portion $\left(22.5^{\circ}\right.$ rotation of main profile or 1/16th part) of flange was modelled with a bolt hole at required position and then reflected symmetrically to complete 360 degree model. Gasket is modelled by rotating an area pattern about $y$-axis through 360 degrees in 16 numbers of volumes; it is possible to model half gasket with respect to thickness due to symmetry of geometry and loading conditions. Bolt is modelled by rotating an area pattern about axis defined by key points through 360 degrees in 4 numbers of volumes and then remaining 7 bolts are generated by virtue of symmetry in $z$-axis; the objective pipe flange connection is tightened by eight bolts. Half portion of bolt was modelled due to plane symmetry of bolt. Only a small portion of pipe is modelled to reduce computational time. The resulted flanged joint model is shown in figure 1a. Commercial FEA software ANSYS (ANSYS 2004) is used during the analysis. A four inch 900\# class, ANSI flange joint is selected for this study.

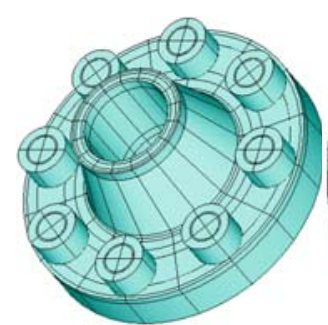

(a)

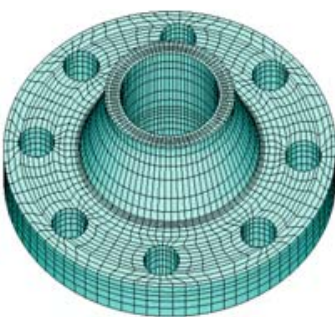

(b)

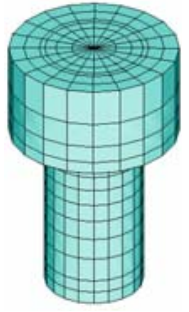

(c)

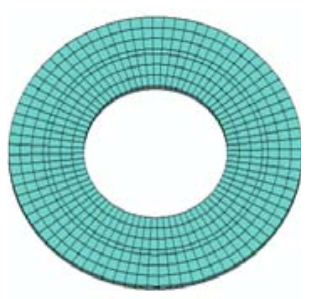

(d)

Figure 1. (a) Full gasketed flanged joint (360 degree); Volumetric Mesh, (b) Flange, (c) Bolt, (d) Gasket. 
Table 1. Material properties.

\begin{tabular}{llccc}
\hline Parts & \multicolumn{1}{c}{ As per code } & E (MPa) & $v$ & Allowable stress (MPa) \\
\hline Flange/Pipe & ASTM A350 LF2 & 173058 & $0 \cdot 3$ & $248 \cdot 2(\sigma y)$ \\
Bolt & ASTM SA193 B7 & 168922 & $0 \cdot 3$ & 723.9 \\
Gasket (solid plate) & ASTM A182 & 164095 & $0 \cdot 3$ & $206 \cdot 8(\sigma y)$ \\
\hline
\end{tabular}

\subsection{Element selection and meshing}

Eight-nodded structural SOLID45 lower order isoperimetric element is used for modelling of flange, bolt, solid gasket and pipe. Three-dimensional 'surface-to-surface' CONTA174 contact elements, in combination with TARGE170 target elements are used between the flange face and gasket, bolt shank and flange hole, the top of the flange and the bottom of the bolt, to simulate contact distribution. Before volume mesh generation, area mesh is created on one side of the flange, bolt and solid plate gasket by specified number of divisions and space ratio for each line. Complete 360-degree flange model mesh is then generated from the angular portion of flange by symmetry reflection for 3-D finite elements as shown in figure $1 \mathrm{~b}$. For bolt and solid plate gasket volumetric mesh is generated by sweeping the mesh from an adjacent area through the volume (figures $1 \mathrm{c}$ and $\mathrm{d}$ ).

\subsection{Material properties}

Allowable stresses and material properties for flange, pipe, and bolt and symmetry plate are given in table 1 (ASME 1998). An elastoplastic material model is used which consists of two sections each having a linear gradient. The first section, which models the elastic material, is valid until the yield stress is reached. The gradient of this section is the Young's modulus of elasticity. The second section which functions beyond the yield stress, and models the behaviour of the plastic material, has a gradient of the plastic tangent modulus, which for this study was $10 \%$ of the Young's modulus of elasticity previously (Abid 2000). Both the elastic and aelasto-plastic material models are used for comparative joint's relxataion behaviour study.

\subsection{Contact generation}

2.4a Between flange, bolt head and gasket: To define contact pair between flange and bolt head, flange face areas are taken as target surface, while bottom areas of the bolt head are taken as contact surface (figure 2a). For contact pair generation between flange bottom surface and gasket (figure 2b), flange bottom surface areas are taken as target surface while gasket top surface areas are taken as contact surface. The contact condition is applied and the friction is taken into consideration at the interfaces between the flange and the gasket. Friction coefficient is varied from $0 \cdot 1$ to $0 \cdot 2$ to prevent rigid motion between flange and gasket surface and its effects on interface stress distributions are examined. However, the effect of friction coefficient is found to be very small.

2.4b Between bolt shank and bolt hole: To prevent rigid motion of flange during bolt up, contact is defined between bolt shanks and the bolt holes in flange (figure 2c), as there is a gap present between the two surfaces. Therefore, contact surface offset (CNOF) is set to adjust initial contact conditions before contact generation. A positive value of $1.61 \mathrm{~mm}$ (gap between 


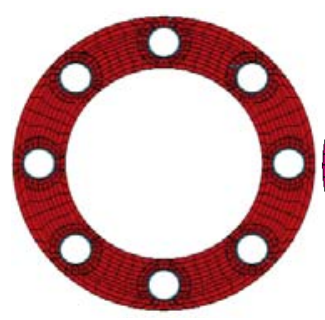

(a)

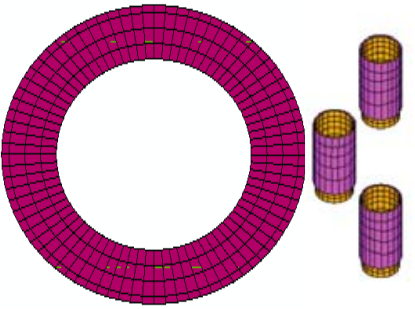

(b)

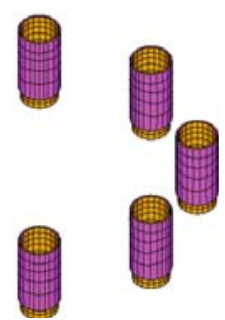

(c)

Figure 2. Contact pair between (a) flange face and bottom of bolt, (b) Flange bottom and Gasket, (c) bolt shank and bolthole.

the two surfaces) is specified to offset the entire contact surface towards the target surface, while a negative value is used to offset the contact surface away from the target surface. In this case, as flange undergoes rigid motion and penetrates into bolt shank, so bolt hole areas in flange are set as contact surface, while bolt shank areas are set to be the target surface.

\subsection{Boundary conditions}

The flange and the gasket are free to move in the axial and the radial direction. Symmetry boundary conditions are applied at the gasket's lower portion. Bolts are constrained in the radial and tangential direction along neutral axis of the bolt. An axial displacement is applied on the bottom area of the bolt shank to get required pre-stress (figure 3a).

2.5a Bolt pre-loading: To ensure a proper joint assembly, the sequence in which bolts are tightened during a pass has a considerable importance. In the present work, following two sequences are used

(i) Sequence 1: 1,5,3,7,2,6,4 and 8 (Abid 2000) (figure 3b)

(ii) Sequence 2: 1,2,3,4,5,6,7 and 8 (Abid 2000) (figure 3c).

Bolts are tightened one by one with the torque control method (Fukuoka \& Takaki 2003). All the bolts are tightened in increments of torques i.e. 210, 310, 400 and $505 \mathrm{Nm}$ as per

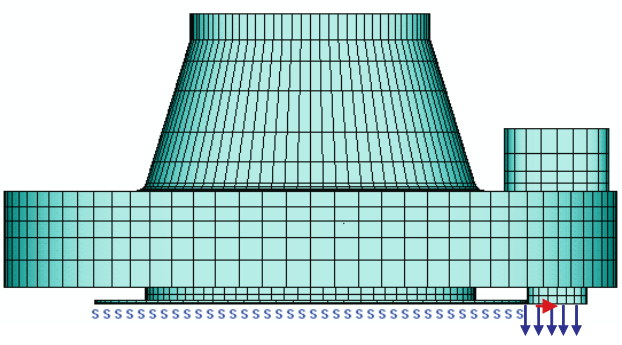

(a)

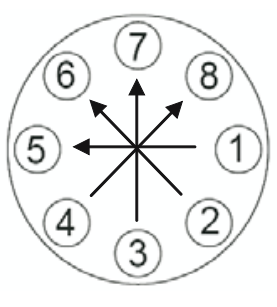

(b)

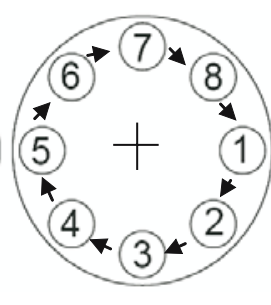

(c)

Figure 3. (a) Boundary conditions; bolt tightening, (b) Sequence 1, (c) Sequence 2. 
Table 2. Target stress calculated for each pass.

\begin{tabular}{lcc}
\hline Applied torque $(\mathrm{Nm})$ & Bolt pre-load $(\mathrm{KN})$ & Target stress $(\mathrm{MPa})$ \\
\hline 210 & 37 & 57 \\
310 & 54 & 86 \\
400 & 70 & 112 \\
505 & 89 & 145 \\
\hline
\end{tabular}

sequence 1 during the first four passes and as per sequence 2 with a torque of $505 \mathrm{Nm}$ in clock-wise direction in one pass. Target torques is converted to the bolt pre-loads for each pass. Using simplified technique given in European Sealing Association (1998), for lubricated fasteners with 0.2 as loss of load factor due to friction, pre-load against given torque (table 2). Average bolt stress is then calculated by dividing the bolt pre-load by the nominal area of bolt shank. The joint is tightened to the target stress for each pass calculated and given in table 2 and using an optimization routine developed. The magnitude of the axial displacement (UY) applied to the bottom area of the bolt shank to pre-stress each bolt to the target stress, is given in table 3. Maximum displacement applied is to achieve $30 \%$ of the yield of the bolt. Although this is considered very low but it avoids gasket crushing, and based on this the maximum recommended applied torque provided by the gasket suppliers is $505 \mathrm{Nm}$ (Abid 2000).

\section{Results and discussions}

\subsection{Bolt stress variation}

To determine bolt relaxation or bolt bending behaviour during tightening the bolts as per sequence 1 and 2, four nodes are selected at an angle of 90 degree on the shank of each bolt. $\mathrm{B} 1 / 1$ and $\mathrm{B} 1 / 2$ represent inner and outer nodes respectively. B1/3 and B1/4 represent side nodes and B1/M represents the mid node on bolt shank. Similar nomenclature is used for all other bolts. For average bolt stress, mid node on the shank of the bolt is selected (figures $4 \mathrm{a}$ and $b$ ).

Figure 4c shows pre-load variation in bolt-1, during other bolt tightening in the joint during pass-1. Stress in bolt-1 reduces, while tightening neighbouring bolt- 2 and bolt- 8 . This is concluded due to the elastic interaction of flange which deforms in axial direction during bolt

Table 3. Magnitude of UY for each pass.

\begin{tabular}{|c|c|c|c|c|c|}
\hline Bolt & Pass-1 & Pass-2 & Pass-3 & Pass-4 & Pass-5 \\
\hline B1 & $0 \cdot 105$ & $0 \cdot 138$ & $0 \cdot 172$ & $0 \cdot 224$ & $0 \cdot 224$ \\
\hline B5 & 0.095 & 0.138 & $0 \cdot 170$ & 0.222 & 0.225 \\
\hline B3 & 0.079 & $0 \cdot 128$ & $0 \cdot 161$ & $0 \cdot 206$ & $0 \cdot 220$ \\
\hline B7 & 0.069 & $0 \cdot 123$ & 0.156 & $0 \cdot 204$ & 0.220 \\
\hline B2 & $0 \cdot 103$ & $0 \cdot 146$ & 0.179 & $0 \cdot 224$ & 0.220 \\
\hline B6 & 0.094 & 0.142 & 0.175 & $0 \cdot 221$ & 0.222 \\
\hline B4 & $0 \cdot 090$ & $0 \cdot 138$ & 0.169 & $0 \cdot 213$ & 0.223 \\
\hline B8 & $0 \cdot 088$ & $0 \cdot 136$ & $0 \cdot 168$ & $0 \cdot 212$ & 0.222 \\
\hline
\end{tabular}




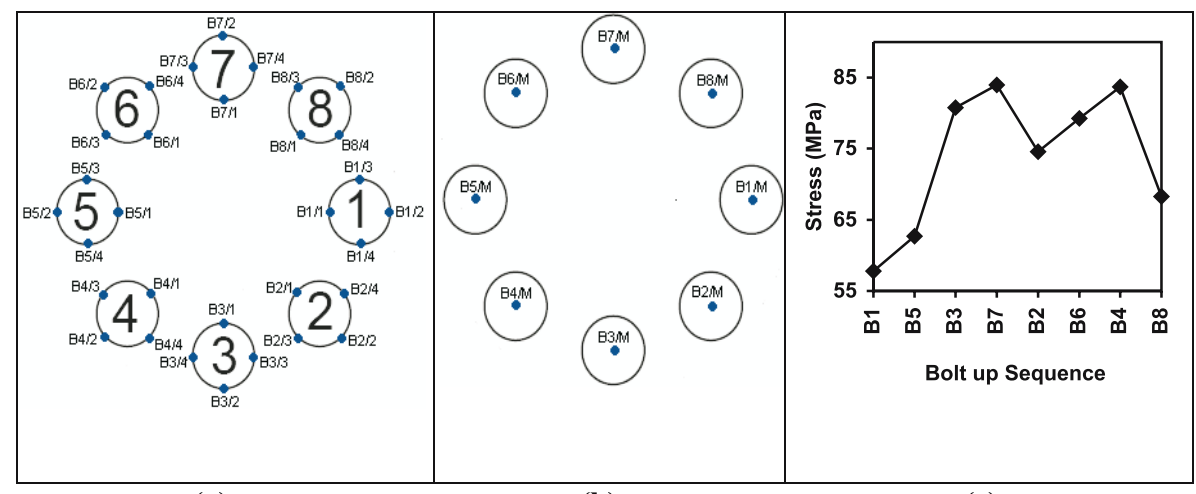

(a)

(b)

(c)

Figure 4. Nomenclature: (a) Side nodes, (b) Mid nodes; (c) Variation of bolt stress of bolt-1 (pass-1).

load application, thus relaxing the bolt closest to the bolt being tightened (Fukuoka $\&$ Takaki 2001). Figure 5a shows relaxation of bolt- 2 and bolt- 4 while tightening bolt- 1 , bolt- 3 and bolt-5. During tightening all other bolts, pre-load of bolt-1 increases and is concluded due to the flange rotation. Figure $5 \mathrm{~b}$ shows increase in stress in bolt-5, while tightening bolt- 1 during pass-1. Almost similar phenomena occurs in all the bolts, however bolt stress variation becomes more uniform with successive passes. Figure 6 shows the effect of tightening of one bolt on all other bolts for pass-1. It is clear that tightening any bolt relaxes its neighbouring bolts while slight rise in stress is observed in the remaining bolts. On tightening the last bolt i.e. bolt- 8 , in pass- $1,27 \%$ to $38 \%$ pre-load relaxation is observed in bolt- 3 and bolt- 7 respectively. Bolt-2,4,6 and 8 remains completely relaxed during tightening bolt-3,5 and 7 . Figure 7 represents the bolt pre-load variation while tightening all the bolts during all the passes as per above mentioned sequences. For all the bolts, almost same stress variation pattern is observed for the first four passes. However, more uniform pre-load distribution is observed during the last pass as per sequence 2. Maximum pre-load variation is observed for bolt-5; as for the first four passes, the maximum stress at bolt- 5 is observed when bolt- 2 is tightened and minimum when bolt- 4 is tightened. The pre-load variations in bolt- 5 during bolt-5 tightening are $29 \mathrm{MPa}$ for the $1^{\text {st }}$ pass, $20 \mathrm{MPa}$ for $2^{\text {nd }}, 17 \mathrm{MPa}$ for $3^{\text {rd }}, 15 \mathrm{MPa}$ for $4^{\text {th }}$ and $4 \mathrm{MPa}$ for the last pass. Bolt- 8 remains relaxed till the end of first pass until it is tightened.

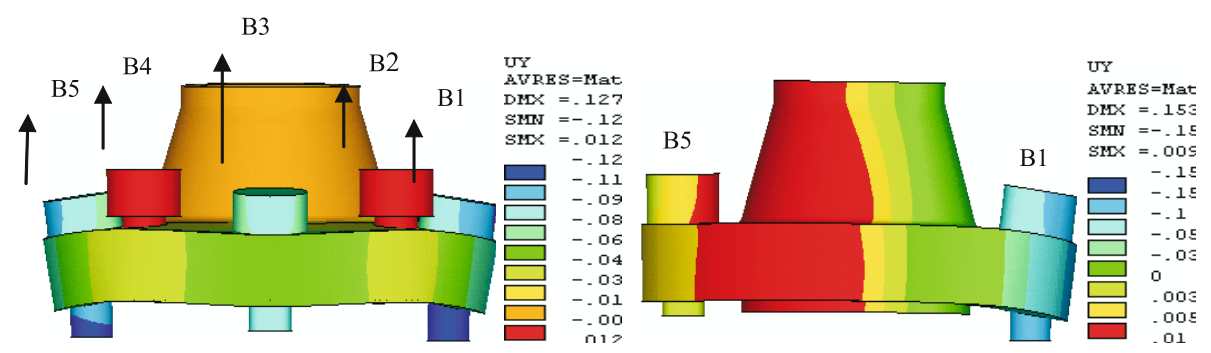

(a)

(b)

Figure 5. Exaggerated deformation plots. (a) Bolt relaxation phenomena, (b) Flange opening phenomena. 


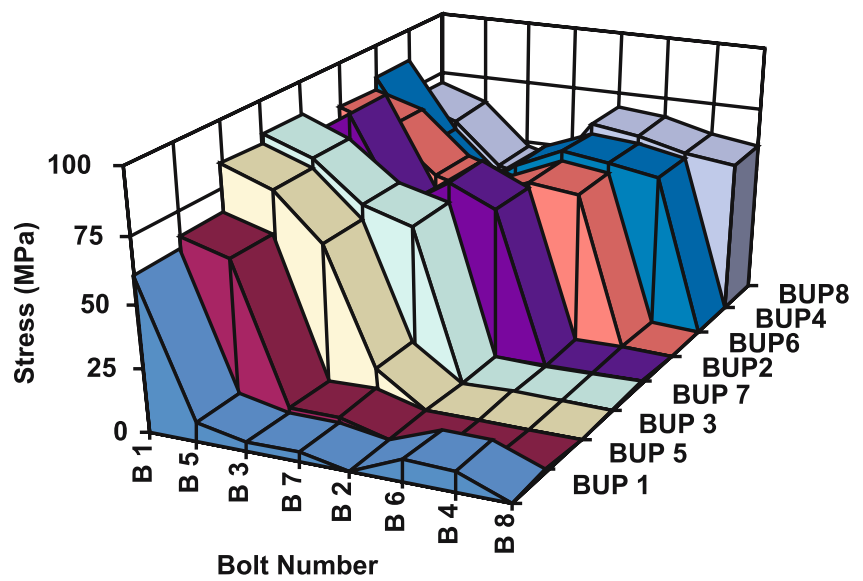

Figure 6. Individual bolt up effect (pass-1).

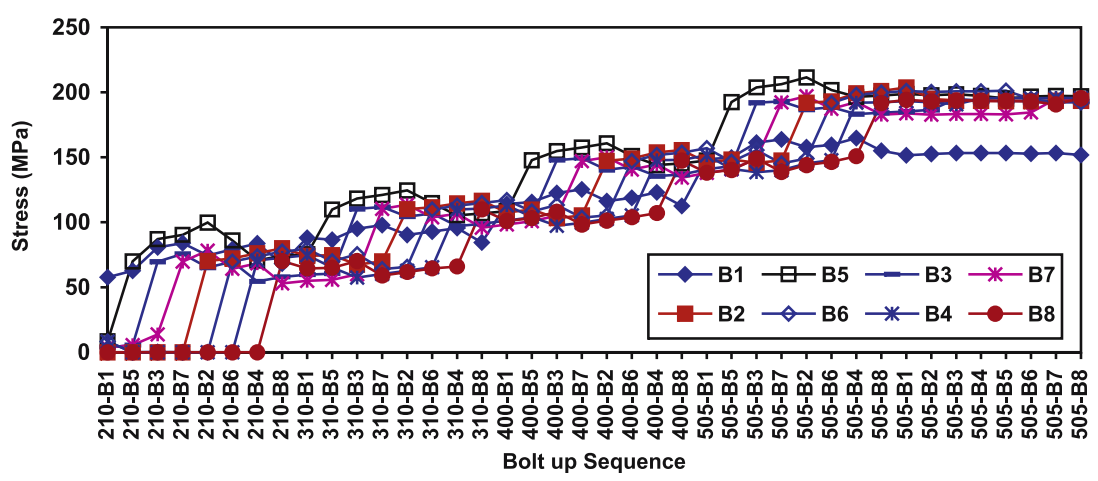

Figure 7. Individual bolt up effect (pass-1).

\subsection{Bolt bending behaviour}

Bolt bending behaviour is studied along four locations along inner, outer and side locations on each bolt shank at the end of each pass (figure 8). Almost similar bending behaviour is observed for all the eight bolts. Tensile stresses in all the bolts are observed at all bolt locations during the bolt up. The difference in axial stresses between the side nodes is negligible for all the bolts, indicating a slight side-wise bolt bending. However, the difference in axial stress between the

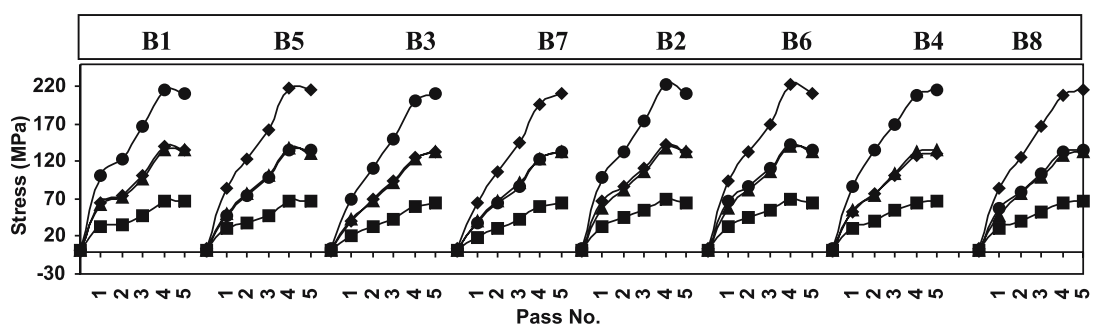

Figure 8. Bolt bending behaviour of all 8 bolts in the joint. 


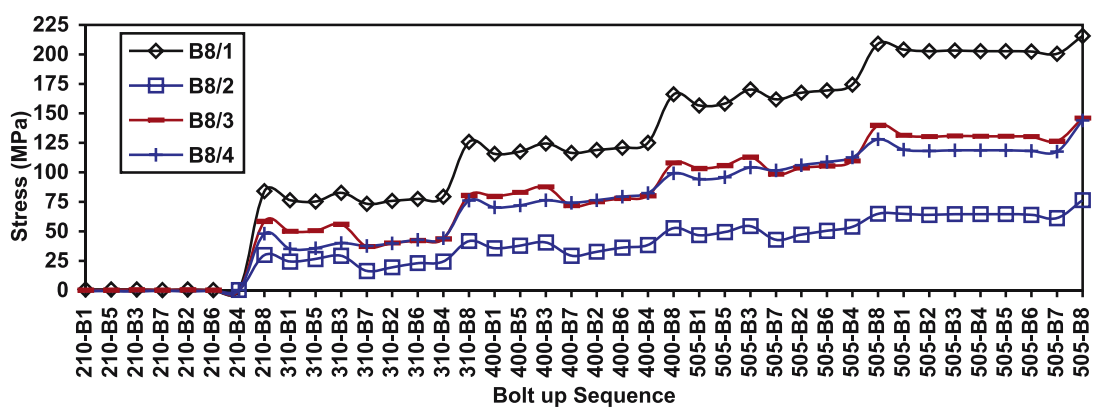

Figure 9. Axial stress variation at the four locations of bolt-8.

inner/outer nodes is obvious. Inner nodes for all the bolts remain in maximum tension while the outer nodes in minimum tension indicating bolt bending. Bending is increased remarkably with increase in torque and is the maximum during the last two passes. It is also observed that during tightening bolts at 90 and 180-degree locations to the target bolt, the difference in axial stresses between side nodes is little but tightening bolts other than at 90 and 180degree locations, the difference becomes dominant; this might be due to flange displacement causing the bolt to bend side-wise. For example, axial stress variations at the four locations of bolt-8 during tightening each bolt are shown in figure 9. During tightening the first four bolts (bolt-1, 3, 5 and 7) in a pass, a slight difference in the axial stress between side nodes is observed, whereas, difference is negligible while tightening the last four bolts in the joint.

\subsection{Bolt scatter}

Figure 10a shows the bolt scatter obtained at the completion of each pass after tightening each bolt to the target stress. Pre-load of bolts 1,3,5 and 7 in the first four passes are less than the target stress. At the end of first pass, 27-38\% pre-load relaxations are observed for bolt-3 and bolt-7 respectively. This is due to the reason that these bolts are tightened in advance of the neighbouring two bolts, so during tightening the last four bolts (bolts-2,4,6 and 8), they undergo relaxation. For example, bolt-7 is found with minimum stress during the first four

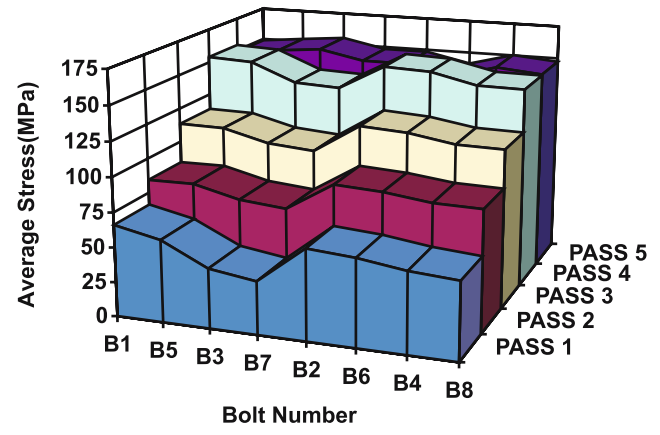

(a)

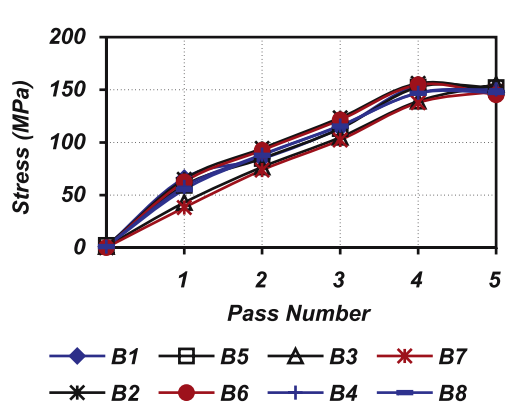

(b)

Figure 10. (a) Scatter in bolt stress at completion of each pass, (b) Bolt stress variation at the end of each pass. 
Table 4. Difference between maximum and minimum bolt stress.

\begin{tabular}{lc}
\hline Pass number & Maximum difference (MPa) \\
\hline 1 & 27 \\
2 & 21 \\
3 & 21 \\
4 & 19 \\
5 & 9 \\
\hline
\end{tabular}

passes, reason is that tightening the last two bolts (bolt-6 and 8) in a given pass, bolt-7 is relaxed. Bolt-2,4,6 and 8 are found at stress level greater than the target stress. The reason being that these bolts are tightened as last four bolts in a pass. Their neighbouring bolts are tightened already, so they have greater stress than the target stress in these bolts. The maximum pre-load difference is observed between bolt- 2 and bolt-7, because bolt- 2 is first bolt after tightening first four bolts. During tightening bolts 4,6 and 8 its pre-load increases, whereas bolt-7 is the last bolt in tightening first four bolts. During tightening bolt- 6 and bolt- 8 at the end of pass, it is relaxed. Scatter is greater in bolts 1,3,5 and 7 as compared to bolts 2,4,6 and 8 because the gasket is seated to its minimum thickness with $1^{\text {st }}$ four bolts tightening. It is observed that the bolt scatter is the maximum for the $1^{\text {st }}$ pass. For second pass, scatter reduces and its magnitude remains almost the same for pass- 3 and pass- 4 and is minimized/reduced at last (5th) pass with sequence 2. This concludes the importance of the last pass with clockwise tightening. Table 4 illustrates difference between the maximum and minimum axial bolt stress. Maximum pre-load difference at the last pass is only $9 \mathrm{MPa}$, ensuring uniform bolt stress distribution.

\subsection{Bolt scatter for elastic model}

Elastic analysis is also performed to investigate the effect of material modelling on bolt scatter and pre-load variations. Same magnitude of UY is applied to the bottom areas of bolt shank as that for elastoplastic model. Boundary conditions, joint configuration, number of tightening passes and their sequence are kept the same to study the comparison of both the material models.

Figure 11a shows the bolt scatter for the elastic model. Almost similar trend is observed for the first four passes with gradual decrease in the scatter. However average stress in each pass for all the bolts are more than the elastoplastic model. Results indicate that pre-load reductions happens for the first four bolts 1,3,5 and 7, whereas bolt- 2 to bolt- 8 are observed with higher stresses than the average. The reason is that bolts 1,5,3 and 7 are tightened in advance of the neighbouring two bolts, unlike the other four bolts.

Figure 11b shows the comparison of the bolt scatter at the end of each pass between elastic and elastoplastic material models. Ratio of bolt stress to the target stress (145 MPa) for each pass is plotted. This is done to obtain the bolt scatter for both the models on same scale (normalized values). Comparing the results, it is noted that the bolt scatter in elastoplastic analysis is more than the elastic analysis because in elastic analysis, stress remains proportional to strain no matter how much the strain increase. Therefore, flange during loading deforms and regains its original shape after load removal, while in the elastoplastic analysis, stress is no more proportional to strain beyond elastic limit, thus flange deforms and do not regain its original shape, hence induced yielding results in increase in scatter. The difference in bolt 


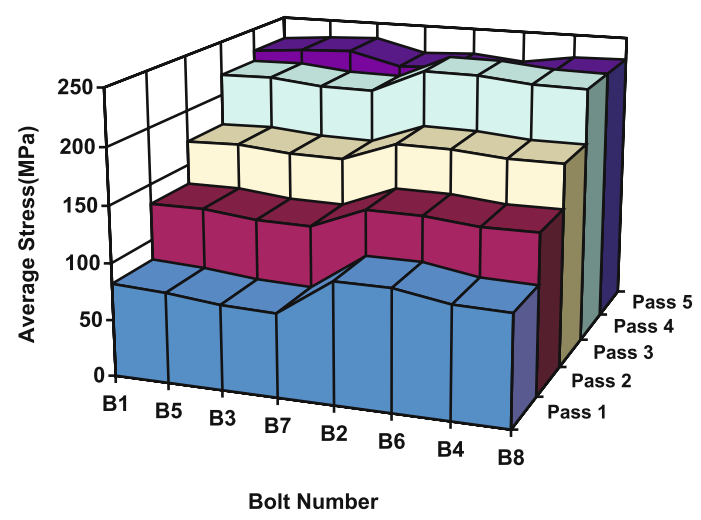

(a)

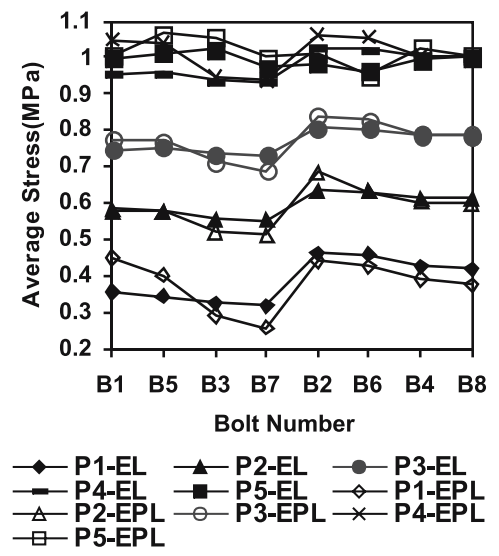

(b)

Figure 11. Scatter in bolt stress at completion of each pass. (a) Elastic model, (b) Elastic and elasto-plastic material models (normalized values).

scatter for both the models is increased at pass- 4 and 5 and elastoplastic model is observed with greater pre-load variations and is possibly due to the yielding initiation at the hub flange fillet.

Fukuoka \& Takaki (2001) performed FE analysis for estimating the scatter in bolt pre-loads when tightening each bolt by torque control method. The author performed elastic analysis and reported the pre-load variations of bolt-1 during pass- 1 and scatter in bolt stress at the completion of bolt up. Present study results for elastic model are in good agreement with the Fukuoka's results. Figure 12a elaborates the pre-load variation of bolt-1 with the bolt up during pass- 1 , whereas figure $12 \mathrm{~b}$ shows the bolt scatter at the end of $1^{\text {st }}$ pass. Elastoplastic material model is used throughout the study unless otherwise mentioned.

\subsection{Gasket stress variation}

One of the main concerns for bolted flange joints is the measure of its sealing capability which is investigated by measuring gasket stress. Nodes on the gasket outer sealing ring as shown

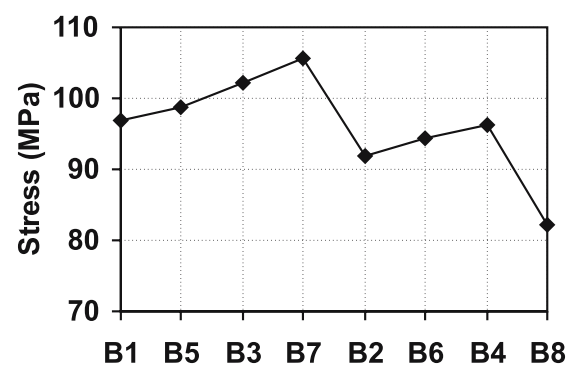

Bolt up Sequence

(a)

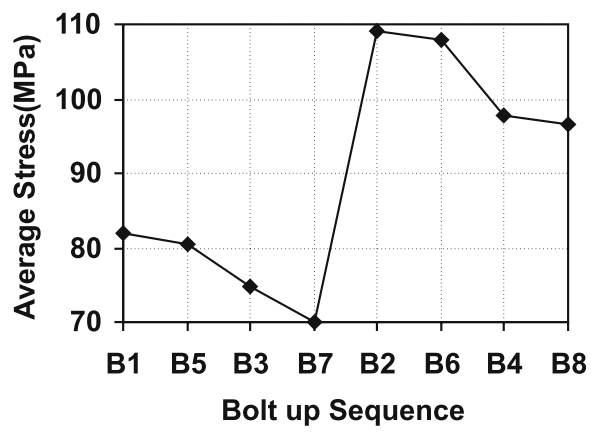

(b)

Figure 12. (a) Variation of bolt stress of bolt-1 during pass-1 (elastic model). (b) Scatter in bolt stress at pass-1 completion (elastic model). 

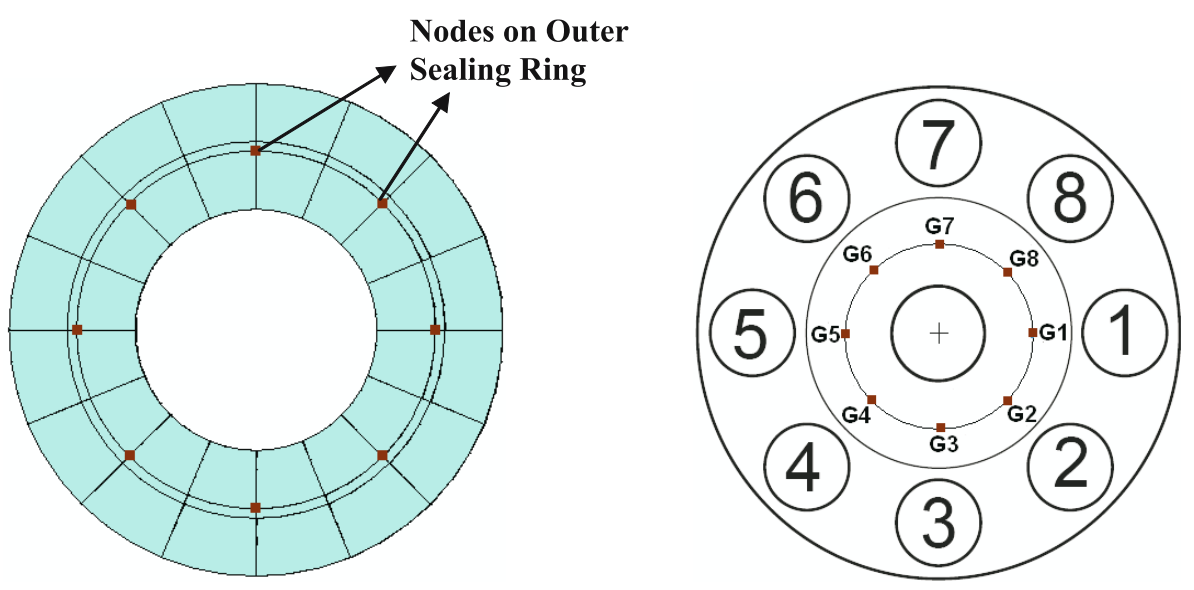

Figure 13. Nomenclature of the selected nodes on gasket.

in figure 13 corresponding to the bolt location are selected to observe contact stress variation with tightening each bolt and observe the effect of bolt scatter on the sealing performance. Figure 14a represents the axial contact stress variation at node closest to bolt-1 (G1) during bolt up. It is observed that during tightening bolt- 1 and its neighbouring bolts, i.e. bolt-2 and bolt- 8 , there is an increase in the stress at G1, whereas during tightening bolt- 4,5 and bolt- 6 , there is a decrease in the magnitude of the stress. Figure $14 \mathrm{~b}$ shows the contact stress distribution along outer sealing ring at the end of each pass, it is seen that for $1^{\text {st }}$ four passes maximum contact stress variation is between G1 (maximum) and G7 (minimum). The contact stress variations along the circumference for the solid plate gasket are not remarkable for any of the pass. Almost uniform distributions are observed ensuring better sealing ability. The difference between the maximum and minimum contact stress along outer sealing ring during all passes is observed in the range of 5 to $13 \mathrm{MPa}$ with maximum after pass- 1 and minimum after pass-5. The minimum required gasket seating stress recommended by the gasket supplier is $68 \mathrm{MPa}$ which is achieved almost after the second pass and is increased to an average contact stress of $110 \mathrm{MPa}$ along all bolt locations with a very slight variation.

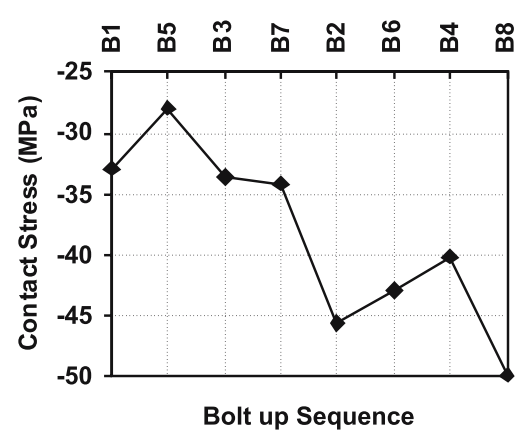

(a)

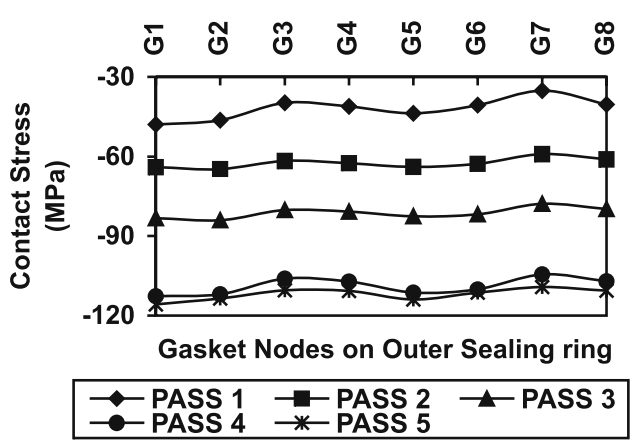

(b)

Figure 14. (a) Axial contact stress variation of G1 with bolt up after tightening pass-1, (b) Contact stress distributions at the end of each pass. 


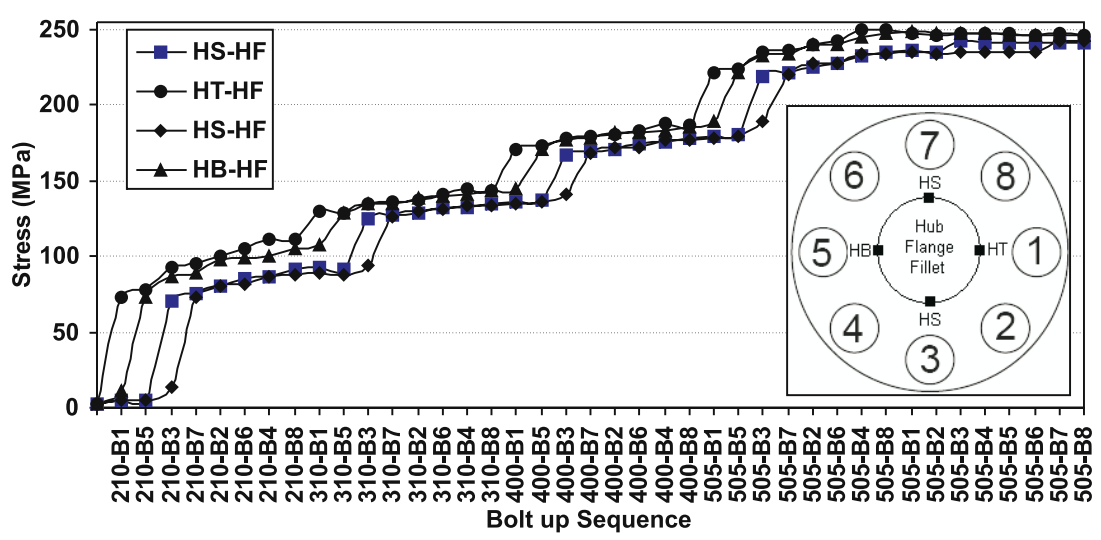

Figure 15. Maximum principal axial stress variation at hub flange fillet during bolt up.

\subsection{Flange stress variation with bolt up}

Figure 15 shows the variation of principal stresses in axial direction at the hub flange fillet. Stress variations observed during tightening first four bolts in a pass are greater than the last four bolts tightening. These variations at the four locations are maximum for the first pass and decrease with each pass and are minimum for the last pass. Maximum scatter is observed during bolt- 5 tightening. Stress is maximum at the location close to the bolt being tightened and minimum at 90-degree locations. Axial stress at hub top and bottom locations is observed greater than side locations. For sequence 1, almost same stress variation pattern observed along all the locations for all torque values $(210-505 \mathrm{Nm})$. Difference between the maximum and minimum axial stresses along hub flange fillet pass during bolt-5 tightening is $72,42,40,45$ and 12 Mpa respectively. Almost uniform stress distribution along hub flange fillet is achieved with the last pass tightening as per tightening sequence 2 .

It is important to note that yielding is observed just to initiate at the last pass at hub top and bottom locations even after utilizing the bolt strength, which is only about 25 to $30 \%$ of the bolt yield.

\section{Conclusions}

From results of study it is concluded that the joint's integrity and sealing performance is very much dependent on the material properties of the gasket used. The joint with solid plate gasket experiences almost uniform bolt stress and bending behaviour and shows almost same variations at all passes. Bolt scatter, bolt bending and bolt relaxation are considered as the main factors affecting the joint's performance. To control these, the use of proper bolt tightening sequence, number of passes are important. A dynamic mode in a gasketed joint is the main reason for its failure. In addition, keeping in view the achieved uniform stress distribution during the entire bolt tightening in the joint along all bolt locations, using a solid plate gasket for a proper joint sealing performance is recommended. It is also concluded that since the elastic model contains no yield criteria, bolt scatter and joint relaxation behaviour cannot be analysed realistically. It is recommended to use an elastoplastic material model for more realistic results. 


\section{References}

Abid M 2000 Experimental and analytical studies of conventional (gasketed) and unconventional (non gasketed) flanged pipe joints (with special emphasis on the engineering of 'joint strength' and 'sealing'). PhD Thesis (Glasgow, UK: University of Strathclyde)

Abid M, Baseer U 2007 3-D Nonlinear finite element analysis of gasketed flange joint under combined internal pressure and different temperatures. J. Eng. Mech. by ASCE. 133/2: 222-229

Abid M, Nash D H 2006 Joint relaxation behaviour of gasketed bolted flanged pipe joint during assembly. $2^{\text {nd }}$ WSEAS International Conference on Applied and Theoretical Mechanics (MECHANICS'06) 319-325

Abid M, Nash D H 2006 Bolt bending behaviour in a bolted flanged pipe joint: A comparative study. ASME Pressure Vessel and Piping Conference, Hyatt Regency Vancouver, Vancouver, British Columbia, Canada, 23-27, 1-8

American Society of Mech. Engrs (ASME) 1998 ASME Boiler and Pressure Vessel Code, Section VIII, New York, USA

ANSYS Inc. 2004 ANSYS Elements Manual, Seventh Edition

European Sealing Association 1998 Guidelines for safe seal usage - Flanges and gaskets. Report No. ESA/FSA 009/98, 1-40

Fukuoka T, Takaki T 2001 Finite element simulation of bolt-up process of pipe flange connections. J. Pressure Vessel Technol. 123: 282-287

Fukuoka T, Takaki T 2003 Finite element simulation of bolt-up process of pipe flange connections with spiral wound gasket. J. Pressure Vessel Technology. 125: 371-377 